\title{
WEIGHT FUNCTIONS IN TIME-FREQUENCY ANALYSIS
}

\author{
KARLHEINZ GRÖCHENIG
}

\begin{abstract}
We discuss the most common types of weight functions in harmonic analysis and how they occur in time-frequency analysis. As a general rule, submultiplicative weights characterize algebra properties, moderate weights characterize module properties, Gelfand-Raikov-Shilov weights determine spectral invariance, and Beurling-Domar weights guarantee the existence of compactly supported test functions.
\end{abstract}

\section{INTRODUCTION}

Weight functions are a very technical topic in time-frequency analysis. Many different conditions on weights appear in the literature, and their motivation is sometimes confusing. This article offers a survey of the most important classes of weight functions in time-frequency analysis.

Weights are used to quantify growth and decay conditions. For instance, if $m(t)=(1+|t|)^{s}$ and $\|f\|_{L_{m}^{\infty}}=\sup _{t \in \mathbb{R}^{d}}|f(t)| m(t)<\infty$, then $|f(t)| \leq C(1+|t|)^{-s}$. So if $s>0$, then this condition describes the polynomial decay of $f$ of order $s$, whereas if $s<0$, then $f$ grows at most like a polynomial of degree $s$. Combining this intuition with $L^{p}$-spaces, one obtains the weighted $L^{p}$-spaces which are defined by the norm $\|f\|_{L_{m}^{p}}=\|f m\|_{p}=\int_{\mathbb{R}^{d}}|f(t)|^{p} m(t)^{p} d t$.

The harmonic analysis of weighted $L^{p}$-spaces is understood to a large extent. An important source for convolution relations and algebra properties is Feichtinger's early paper [11].

Weight functions in time-frequency analysis occur in many problems and contexts: (a) in the definition of modulation spaces where the weight helps to measure and describe the time-frequency concentration of a function, (b) in the definition of symbol classes for pseudodifferential operators where the weight describes the specific form of smoothness in the Sjöstrand class, and (c) in the theory of Gabor frames and time-frequency expansions where the weight measures the quality of time-frequency concentration.

This article is organized as follows: Section 2 contains the definitions and examples of several classes of weight functions, Section 3 the main definitions of time-frequency analysis. In the subsequent sections we discuss the class of submultiplicative weights, moderate weights, GRS-weights, subconvolutive weights, and Beurling-Domar weights. In each section, we recall first the main definition and

Key words and phrases. Weight function, submultiplicative, moderate, subconvolutive, Beurling-Domar condition, GRS-condition, Gabor frame, modulation space, pseudodifferential operator, symbol class, Wiener's Lemma.

K. G. was supported by the Marie-Curie Excellence Grant MEXT-CT 2004-517154. 
then state the characterizing property in the context of $L^{p}$-spaces. We then present the main applications and results about these weights in time-frequency analysis. Although we will not offer complete proofs, we try to sketch those proof ideas that shed some light on why a particular weight class arises in harmonic analysis and time-frequency analysis.

\section{Classes of Weight Functions}

In general a weight function $m$ on $\mathbb{R}^{d}$ is simply a non-negative function. We will assume without loss of generality that the weight is continuous.

In time-frequency analysis the following types of weight functions occur.

Definition 1. Let $v$ and $m$ be non-negative functions on $\mathbb{R}^{d}$ or on $\mathbb{Z}^{d}$.

(a) A weight $v$ is called submultivplicative, if

$$
v(x+y) \leq v(x) v(y) \quad \forall x, y \in \mathbb{R}^{d} .
$$

(b) Given a submultiplicative weight $v$, a non-negative function $m$ is called a $v$-moderate weight, if there exists a constant $C>0$, such that

$$
m(x+y) \leq C v(x) m(y) \quad \forall x, y \in \mathbb{R}^{d} .
$$

We denote the set of all $v$-moderate weights by $\mathcal{M}_{v}$. If $m$ is $v$-moderate with respect to some $v$, then we simply call $m$ moderate.

(c) A non-negative function $v$ is called subconvolutive, if $v^{-1} \in L^{1}\left(\mathbb{R}^{d}\right)$ and $v^{-1} *$ $v^{-1} \leq C v^{-1}$ (as a pointwise inequality).

(d) A submultiplicative weight $v$ satisfies the GRS-condition (the Gelfand-RaikovShilov condition), if

$$
\lim _{n \rightarrow \infty} v(n x)^{1 / n}=1 \quad \forall x \in \mathbb{R}^{d} .
$$

Equivalently, $\lim _{n \rightarrow \infty} \log v(n x) / n=0$ for all $x \in \mathbb{R}^{d}$.

(e) A submultiplicative weight $v$ satisfies the Beurling-Domar condition (BDcondition), if

$$
\sum_{n=0}^{\infty} \frac{\log v(n x)}{n^{2}}<\infty \quad \forall x \in \mathbb{R}^{d} .
$$

(f) A submultiplicative weight $v$ satisfies the logarithmic integral condition, if

$$
\int_{|x| \geq 1} \frac{\log v(x)}{|x|^{d+1}} d x<\infty
$$

REMARKS: 1. All these conditions (except for (f)) make sense on arbitrary locally compact Abelian groups or even on more general groups, but for simplicity we will restrict our attention to $\mathbb{R}^{d}$, the corresponding phase-space $\mathbb{R}^{2 d}$, and to $\mathbb{Z}^{d}$.

2. Feichtinger's early paper [11] contains a detailed study of submultiplicative, moderate, and subconvolutive weights on locally compact groups with many examples and counter-examples. GRS-weights occur first in the Russian literature [14]. The logarithmic integral condition was found by Beurling [4], it is so prominent in 
analysis that Koosis devoted an entire monograph to it [31], the Beurling-Domar condition was discovered by Domar in [9].

The standard examples. We consider the following class of weight functions:

$$
m(x)=m_{a, b, s, t}(x)=e^{a|x|^{b}}(1+|x|)^{s}(\log (e+|x|))^{t} .
$$

In particular, this class contains

- the polynomial weights $m_{s}(x)=(1+|x|)^{s}, s \in \mathbb{R}$,

- the exponential weights $m(x)=e^{a|x|}, a \in \mathbb{R}$,

- and the subexponential weights $m(x)=e^{a|x|^{b}}$ for $0 \leq b<1$ and $a \in \mathbb{R}$.

The following classification is taken from [11]. The proof is elementary and omitted.

Lemma 2.1. (a) If $a, s, t \geq 0$ and $0 \leq b \leq 1$, then $m=m_{a, b, s, t}$ is submultiplicative.

(b) If $a, s, t \in \mathbb{R}$ and $0 \leq b \leq 1$, then $m=m_{a, b, s, t}$ is moderate.

(c) If either $0<b<1, a>0, s, t \in \mathbb{R}$ or $b \in\{0,1\}$ and $s>d$, then $m_{a, b, s, t}$ is subconvolutive.

(d) If $a, s, t \geq 0$ and $0 \leq b<1$, then $m_{a, b, s, t}$ satisfies the GRS-condition, the Beurling-Domar condition and the logarithmic integral condition.

Lemma 2.2. If $v$ satisfies the Beurling-Domar condition, then it satisfies the GRScondition.

Proof. We argue by contradiction and show that if the GRS-condition is violated, then the Beurling-Domar condition cannot hold either. Since $\log v\left(x^{n}\right)$ is subadditive, a standard lemma [10, Lemma VIII.1.4] implies that

$$
\lim _{n \rightarrow \infty} \frac{\log v\left(x^{n}\right)}{n}=\inf _{n \rightarrow \infty} \frac{\log v\left(x^{n}\right)}{n} .
$$

In particular, this limit exists. If the GRS-condition is not satisfied, then there exists a subsequence $n_{k}$ and $\alpha>0$, such that $\frac{\log v\left(x^{n}\right)}{n} \geq \alpha>0$ and by (7) therefore $\frac{\log v\left(x^{n}\right)}{n} \geq \frac{\alpha}{2}>0$ for all $n \geq N_{0}$. Consequently we also have $\sum_{n=N_{0}}^{\infty} \frac{\log v\left(x^{n}\right)}{n^{2}} \geq$ $\sum_{n=N_{0}}^{\infty} \frac{\alpha}{2 n}=\infty$. So the Beurling-Domar condition is not satisfied.

REMARKS: 1 . The weight $v(x)=e^{|x| / \log (e+|x|)}$ is submultiplicative; it satisfies the GRS-condition, but not the BD-condition.

2. It is known that the Beurling-Domar condition and the logarithmic integral condition are equivalent.

3. Other examples of weights can be obtained by replacing the Euclidean norm $|\cdot|$ on $\mathbb{R}^{d}$ by some other norm on $\mathbb{R}^{d}$ and by restriction to a subspace of $\mathbb{R}^{d}$. As an example of this procedure we mention $m\left(x_{1}, x_{2}, x_{3}\right)=\left(1+\left|x_{1}\right|^{p}+\left|x_{3}\right|^{q}\right)^{s}$ on $\mathbb{R}^{3}$ where $1 \leq p, q<\infty$ and $s \in \mathbb{R}$. 


\section{The Short-Time Fourier Transform}

If $X=(x, \xi) \in \mathbb{R}^{2 d}$ is a point in the time-frequency plane, the corresponding time-frequency shift is defined by

$$
\pi(X) f(t)=M_{\xi} T_{x} f(t)=e^{2 \pi i \xi \cdot t} f(t-x) \quad t \in \mathbb{R}^{d} .
$$

Time-frequency shifts do not commute, instead they satisfy the canonical commutation relations

$$
M_{\xi} T_{x}=e^{2 \pi i x \cdot \xi} T_{x} M_{\xi} \quad(x, \xi) \in \mathbb{R}^{2 d} .
$$

The transform associated to time-frequency shifts is the short-time Fourier transform. Let $g$ be a suitable non-zero window function on $\mathbb{R}^{d}$, then the short-time Fourier transform (STFT) of a function or distribution $f$ is defined to be

$$
\begin{aligned}
V_{g} f(X) & =\int_{\mathbb{R}^{d}} f(t) \overline{g(t-x)} e^{-2 \pi i \xi \cdot t} d t \\
& =\langle f, \pi(X) g\rangle=\left\langle f, M_{\xi} T_{x} g\right\rangle .
\end{aligned}
$$

The STFT is well defined when we take $g$ to be in a space of test functions $\mathcal{X}$ that is invariant under time-frequency shifts and $f$ in the dual space $\mathcal{X}^{\prime}$. For instance, we may take $f, g \in L^{2}\left(\mathbb{R}^{d}\right)$; or $g \in \mathcal{S}\left(\mathbb{R}^{d}\right)$ (the Schwartz class) and $f \in \mathcal{S}^{\prime}\left(\mathbb{R}^{d}\right)$ (the tempered distributions). No matter which choice of test functions we make, the Gaussian $\varphi(t)=e^{-\pi t \cdot t}$ will always work as a suitable window.

The covariance property says that time-frequency shifts are mapped to shifts in the time-frequency plane, because

$$
\left|V_{g}(\pi(Y) f)(X)\right|=\left|V_{g} f(X-Y)\right| .
$$

For a detailed discussion of time-frequency shifts and the short-time Fourier transform we refer to [15, Ch. 3].

\section{Submultiplicative Weights}

Recall that a non-negative function $v$ on $\mathbb{R}^{d}$ is submultiplicative, if

$$
v(x+y) \leq v(x) v(y) \quad \forall x, y \in \mathbb{R}^{d} .
$$

Submultiplicative weights characterize (Banach) algebra properties.

The standard property in harmonic analysis. As usual, $L_{v}^{1}\left(\mathbb{R}^{d}\right)$ is the Banach space defined by the norm

$$
\|f\|_{L_{v}^{1}}:=\int_{\mathbb{R}^{d}}|f(t)| v(t) d t=\|f v\|_{1} .
$$

Likewise, $\ell_{v}^{1}\left(\mathbb{Z}^{d}\right)$ is defined by the norm $\|\mathbf{a}\|_{\ell_{v}^{1}}=\sum_{k \in \mathbb{Z}^{d}}\left|a_{k}\right| v(k)$.

Lemma 4.1. The space $\ell_{v}^{1}\left(\mathbb{Z}^{d}\right)$ is a Banach algebra under convolution, if and only if $v$ is submultiplicative on $\mathbb{Z}^{d}$, and $L_{v}^{1}\left(\mathbb{R}^{d}\right)$ is a Banach algebra under convolution, if and only if $v$ is submultiplicative on $\mathbb{R}^{d}$. 
Proof. We use $v(l) \leq v(k) v(l-k)$ and estimate in a straightforward manner:

$$
\begin{aligned}
\|\mathbf{a} * \mathbf{b}\|_{\ell_{v}^{1}} & =\sum_{l \in \mathbb{Z}^{d}}\left|\sum_{k \in \mathbb{Z}^{d}} a_{k} b_{l-k}\right| v(l) \\
& \leq \sum_{l \in \mathbb{Z}^{d}} \sum_{k \in \mathbb{Z}^{d}}\left|a_{k}\right| v(k)\left|b_{l-k}\right| v(l-k) \\
& =\left(\sum_{l \in \mathbb{Z}^{d}}\left|b_{l}\right| v(l)\right)\left(\sum_{k \in \mathbb{Z}^{d}}\left|a_{k}\right| v(k)\right)=\|\mathbf{a}\|_{\ell_{v}}\|\mathbf{b}\|_{\ell_{v}} .
\end{aligned}
$$

To show the converse, let $\delta_{m}(k)=1$ for $k=m$ and $\delta_{m}(k)=0$ for $k \neq m$. Then $\delta_{m} * \delta_{n}=\delta_{m+n}$ and $\left\|\delta_{m}\right\|_{\ell_{v}^{1}}=v(m)$. Thus, if $\ell_{v}^{1}$ is a Banach algebra, then

$$
v(m+n)=\left\|\delta_{m+n}\right\|_{\ell_{v}^{1}}=\left\|\delta_{m} * \delta_{n}\right\|_{\ell_{v}^{1}} \leq\left\|\delta_{m}\right\|_{\ell_{v}^{1}}\left\|\delta_{n}\right\|_{\ell_{v}^{1}}=v(m) v(n),
$$

and so $v$ is submultiplicative. The proof for $L_{v}^{1}\left(\mathbb{R}^{d}\right)$ is similar, but requires approximate identities for the converse.

REMARKS: 1 . Usually $L_{v}^{1}\left(\mathbb{R}^{d}\right)$ is equipped with the involution $f^{*}(x):=\overline{f(-x)}$. It is easy to verify that this involution is continuous, if and only if $v(-x) \leq C v(x)$. It is therefore convenient, but not absolutely necessary, to assume that $v$ is an even function. If $v$ is even, then $v(0) \leq v(0)^{2} \leq v(x) v(-x)=v(x)^{2}$. Thus either $v(0)=0$ and $v \equiv 0$ (because $v(x) \leq v(0) v(x)$ ), or $v(0) \geq 1$ and then $v(x) \geq 1$ for all $x$.

2. From now on, we will assume without loss of generality that $v$ is an even function, because we are mostly interested in involutive Banach algebras. The treatment of Banach algebras with unbounded involution is also possible [35], but this case occurs rarely in time-frequency analysis.

Lemma 4.2. If $v$ is submultiplicative (and even), then there exists a constant $a \geq 0$, such that

$$
v(x) \leq e^{a|x|} .
$$

Every submultiplicative weight grows at most exponentially.

Proof. Define $a$ by $e^{a}=\sup _{|t| \leq 1} v(t)$. Since $v$ is continuous and $1=v(0) \leq$ $v(x) v(-x)$, we have $a \geq 1$. Given $x \in \mathbb{R}^{d}$ arbitrary, choose $n \in \mathbb{N}$, so that $n-1<|x| \leq n$. Then $|x / n| \leq 1$ and by the submultiplicativity we find that

$$
v(x)=v\left(n \frac{x}{n}\right) \leq v\left(\frac{x}{n}\right)^{n} \leq e^{a n},
$$

thus $v$ grow at most exponentially.

In time-frequency analysis submultiplicative weights occur in the investigation of twisted convolution, in the definition of "good windows" and spaces of test functions, and in the construction of algebras of pseudodifferential operators. 
4.1. Series of Time-Frequency Shifts and Twisted Convolution. Given a lattice $\Lambda=\alpha \mathbb{Z}^{d} \times \beta \mathbb{Z}^{d} \subseteq \mathbb{R}^{2 d}$, we consider series of time-frequency shifts $A=\sum_{k, l \in \mathbb{Z}^{d}} a_{k l} M_{\beta l} T_{\alpha k}$. To avoid convergence problems, it is often convenient to consider absolutely convergent series of time-frequency shifts. This motivates the following definition.

Definition 2. The linear space $\mathcal{A}_{v}(\alpha, \beta)$ consists of all series of time-frequency shifts $A=\sum_{k, l \in \mathbb{Z}^{d}} a_{k l} M_{\beta l} T_{\alpha k}$ with $\mathbf{a}=\left(a_{k l}\right)_{k, l \in \mathbb{Z}^{d}} \in \ell_{v}^{1}\left(\mathbb{Z}^{2 d}\right)$.

Let $\pi(\mathbf{a})=\sum_{k, l \in \mathbb{Z}^{d}} a_{k l} M_{\beta l} T_{\alpha k}$ be mapping from coefficients to operators. By definition $\pi$ maps $\ell_{v}^{1}\left(\mathbb{Z}^{2 d}\right)$ onto $\mathcal{A}_{v}(\alpha, \beta)$. It can be shown that $\pi$ is one-to-one $[19$, 38]. Consequently $\|A\|_{\mathcal{A}_{v}}:=\|\mathbf{a}\|_{\ell_{v}^{1}}$ is a Banach space norm on $\mathcal{A}_{v}(\alpha, \beta)$.

Lemma 4.3. If $v$ is submultiplicative, then $\mathcal{A}_{v}(\alpha, \beta)$ is a Banach algebra.

Proof. We introduce a new product between two sequences $\mathbf{a}$ and $\mathbf{b}$ : the twisted convolution is defined by

$$
(\mathbf{a} \sharp \mathbf{b})(k, l)=\sum_{k^{\prime}, l^{\prime} \in \mathbb{Z}^{d}} a_{k^{\prime} l^{\prime}} b_{k-k^{\prime}, l-l^{\prime}} e^{-2 \pi i \theta k^{\prime} \cdot\left(l-l^{\prime}\right)} .
$$

Then a simple computation using the commutation relations (9) shows that the composition of two series of time-frequency shifts corresponds to the twisted convolution of the coefficient sequences. Formally, if $A=\pi(\mathbf{a})$ and $B=\pi(\mathbf{b})$, then

$$
A B=\pi(\mathbf{a}) \pi(\mathbf{b})=\pi(\mathbf{a} \sharp \mathbf{b}) .
$$

Twisted convolution is majorized by ordinary convolution via the obvious pointwise inequality

$$
|(\mathbf{a} \sharp \mathbf{b})(k, l)| \leq(|\mathbf{a}| *|\mathbf{b}|)(k, l), \quad \forall k, l \in \mathbb{Z}^{d} .
$$

Therefore the Banach algebra property of $\ell_{v}^{1}\left(\mathbb{Z}^{2 d}\right)$ implies that

$$
\|A B\|_{\mathcal{A}_{v}}=\|\pi(\mathbf{a} \sharp \mathbf{b})\|_{\mathcal{A}_{v}}=\|\mathbf{a} \sharp \mathbf{b}\|_{\ell_{v}^{1}} \leq\|\mathbf{a}\|_{\ell_{v}^{1}}\|\mathbf{b}\|_{\ell_{v}^{1}}=\|A\|_{\mathcal{A}_{v}}\|B\|_{\mathcal{A}_{v}} .
$$

So $\mathcal{A}_{v}$ is a Banach algebra.

REMARK: A similar statement holds for series of time-frequency shifts on an arbitrary lattice.

4.2. Spaces of Test Functions and "Good" Windows. In analysis, test functions are defined by their smoothness and decay conditions, and the resulting spaces are usually Frechet spaces. In time-frequency analysis, the appropriate spaces of test functions are defined by properties of the STFT.

Definition 3. Let $\varphi(t)=e^{-\pi t^{2}}$ be the Gaussian and $v$ be a submultiplicative weight on $\mathbb{R}^{2 d}$. The modulation space $M_{v}^{1}\left(\mathbb{R}^{d}\right)$ consists of all $f \in L^{2}\left(\mathbb{R}^{d}\right)$ for which the norm

$$
\|f\|_{M_{v}^{1}}=\int_{\mathbb{R}^{2 d}}\left|V_{\varphi} f(X)\right| v(X) d X
$$

is finite. 
The following lemma asserts that $M_{v}^{1}$ is always non-trivial.

Lemma 4.4. If $v$ is submultiplicative, then $M_{v}^{1}$ is non-trivial. Specifically, if $\varphi(t)=e^{-\pi t^{2}}$, then $V_{\varphi} \varphi \in M_{v}^{1}$. More generally, every function of the form $g=$ $\sum_{j=1}^{n} c_{j} \pi\left(X_{j}\right) \varphi$, where $c_{j} \in \mathbb{C}, X_{j} \in \mathbb{R}^{2 d}$, belongs to $M_{v}^{1}$.

Proof. A calculation with Gaussian integrals shows that $V_{\varphi} \varphi(X)=2^{-d / 2} e^{i \pi x \cdot \xi} e^{-\pi X^{2} / 2}[15$, Lemma 1.5.1]. Since $v$ grows at most exponentially by Lemma 4.2, we find that $V_{\varphi} \varphi \in L_{v}^{1}\left(\mathbb{R}^{2 d}\right)$ and thus $\varphi \in M_{v}^{1}$ for an arbitary submultiplicative weight $v$.

If $g=\sum_{j=1}^{n} c_{j} \pi\left(X_{j}\right) \varphi$, then $\left|V_{\varphi} g(X)\right| \leq \sum_{j=1}^{n}\left|c_{j}\right|\left|V_{\varphi} \varphi\left(X-X_{j}\right)\right|$ after using (11). We obtain

$$
\|g\|_{M_{v}^{1}}=\left\|V_{\varphi} g\right\|_{L_{v}^{1}} \leq \sum_{j=1}^{n}\left|c_{j}\right|\left\|V_{\varphi} \varphi\left(\cdot-X_{j}\right)\right\|_{L_{v}^{1}} \leq \sum_{j=1}^{n}\left|c_{j}\right| v\left(X_{j}\right)\left\|V_{\varphi} \varphi\right\|_{L_{v}^{1}}<\infty .
$$

So $g \in M_{v}^{1}$.

4.3. Symbol Classes for Pseudodifferential Operators. We consider pseudodifferential operators in the Kohn-Nirenberg correspondence. Given a symbol $\sigma$ on $\mathbb{R}^{2 d}$, the pseudodifferential operator $K_{\sigma}$ is defined by

$$
K_{\sigma} f(t)=\int_{\mathbb{R}^{d}} \sigma(x, \xi) \hat{f}(\xi) e^{2 \pi i x \cdot \xi} d \xi,
$$

whenever the integral makes sense. In the tradition of PDE, this operator is written as $\sigma(x, D)$. Using a suitable duality, the Kohn-Nirenberg correspondence can be defined for an arbitrary tempered distribution $\sigma \in \mathcal{S}^{\prime}\left(\mathbb{R}^{2 d}\right)$, and for even more general distribution classes.

After the fundamental papers of J. Sjöstrand [40,41], the following symbol classes have gained some prominence in the investigation of pseudodifferential operators.

Definition 4. Let $v$ be a submultiplicative (and even) weight function on $\mathbb{R}^{2 d}$ and $\Phi(X)=e^{-\pi X^{2}}$. The weighted Sjöstrand class $M_{v}^{\infty, 1}\left(\mathbb{R}^{2 d}\right)$ is defined by the norm

$$
\|\sigma\|_{M_{v}^{\infty, 1}}=\int_{\mathbb{R}^{2 d}} \sup _{X \in \mathbb{R}^{2 d}}\left|V_{\Phi} \sigma(X, \Xi)\right| v(\Xi) d \Xi
$$

Then $M_{v}^{\infty, 1}$ is a subspace of $\mathcal{S}^{\prime}\left(\mathbb{R}^{2 d}\right)$, consisting of bounded function that are locally in the Fourier algebra.

Composition of pseudodifferential operators defines a product on the level of symbols as follows

$$
K_{\sigma \circ \tau}=K_{\sigma} K_{\tau}
$$

If $\sigma, \tau \in \mathcal{S}\left(\mathbb{R}^{2 d}\right)$, then the product is given by the explicit formula [27]

$$
(\sigma \circ \tau)(x, \xi)=\int_{\mathbb{R}^{2 d}} \sigma(y, \xi+\eta) \tau(x+y, \xi) e^{-2 \pi i y \cdot \eta} d y d \eta .
$$


REMARK: There are many other calculi of pseudodifferential operators, the most important one is the Weyl calculus. The results discussed in this survey are independent of the used calculus. We use the Kohn-Nirenberg correspondence, because it is universal and can be formulated on arbitrary locally compact abelian groups.

One of the key properties of the Sjöstrand classes is the algebra property. The following theorem was proved in [40] for the unweighted case $v \equiv 1$, a different proof was given in [42], weighted versions and two genuine time-frequency proofs were obtained in $[17,18]$ (for the Weyl calculus), the extension to LCA groups is contained in [23].

Theorem 4.5. If $v$ is submultiplicative on $\mathbb{R}^{2 d}$, then $M_{v}^{\infty, 1}\left(\mathbb{R}^{2 d}\right)$ is a Banach algebra with respect to the product $\circ$. Furthermore,

$$
\|\sigma \circ \tau\|_{M_{v}^{\infty, 1}} \leq C_{\Phi}\|\sigma\|_{M_{v}^{\infty, 1}}\|\tau\|_{M_{v}^{\infty, 1}} \quad \forall \sigma, \tau \in M_{v}^{\infty, 1}\left(\mathbb{R}^{2 d}\right) .
$$

Proof. We sketch the proof as it is given in [17]. We define the "grand symbol" of $K_{\sigma}$ by

$$
G(\sigma)(\Xi)=\sup _{X \in \mathbb{R}^{2 d}}\left|V_{\Phi} \sigma(X, \Xi)\right|
$$

Then $\|\sigma\|_{M_{v}^{\infty, 1}}=\|G(\sigma)\|_{L_{v}^{1}}$ by definition of $M_{v}^{\infty, 1}$. The technical and difficult part of the proof is to show the pointwise inequality

$$
G(\sigma \circ \tau)(\Xi) \leq(G(\sigma) * G(\tau) * H)(\Xi) \quad \forall \Xi \in \mathbb{R}^{2 d},
$$

where $*$ is the ordinary convolution on $\mathbb{R}^{2 d}$ and $H$ is a positive function depending only on $\Phi$. Now the algebra property of $M_{v}^{\infty, 1}$ follows from the algebra property of $L_{v}^{1}\left(\mathbb{R}^{2 d}\right)$ stated in Lemma 4.1, because

$$
\begin{aligned}
\|\sigma \circ \tau\|_{M_{v}^{\infty, 1}} & =\|G(\sigma \circ \tau)\|_{L_{v}^{1}} \\
& \leq\|G(\sigma) * G(\tau) * H\|_{L_{v}^{1}} \\
& \leq\|G(\sigma)\|_{L_{v}^{1}}\|G(\tau)\|_{L_{v}^{1}}\|H\|_{L_{v}^{1}} \\
& =C\|\sigma\|_{M_{v}^{\infty, 1}}\|\tau\|_{M_{v}^{\infty, 1}}
\end{aligned}
$$

REMARK: Let us emphasize that the above result works for arbitrary submultiplicative weights, including exponential weights.

\section{Moderate Weights}

Moderate weights comprise a more general class of weight functions and are always associated to a submultiplicative weight $v$. Precisely, a non-negative function is called $v$-moderate, if

$$
m(x+y) \leq C v(x) m(y) \quad \forall x, y \in \mathbb{R}^{d} .
$$


We write $\mathcal{M}_{v}$ for the class of all $v$-moderate weight functions. From this definition follows that

$$
\frac{1}{C v(x)} \leq m(x) \leq C v(x)
$$

and so a moderate weight can grow only as fast as the associated submultiplicative weight $v$. Furthermore, if $K \subseteq \mathbb{R}^{d}$ is compact, then $\sup _{t \in K} m(x+t) \leq$ $C \sup _{t \in K} v(t) m(x)=C^{\prime} m(x)$ and likewise $\inf _{t \in K} m(x+t) \geq C^{\prime \prime} m(x)$.

Alternatively, a non-negative function is moderate, if and only if

$$
\sup _{y \in \mathbb{R}^{d}} \frac{m(x+y)}{m(y)}=C(x)<\infty \quad \forall x \in \mathbb{R}^{d} .
$$

This definition does not make reference to a submultiplicative weight $v$. A related condition occurs in $[26,18.4 .2]$.

$$
\text { Moderate weights arise in "module properties". }
$$

Given a weight $m$, we define the weighted $L^{p}$-space $L_{m}^{p}$ by the norm

$$
\|f\|_{L_{m}^{p}}:=\|f m\|_{p}=\left(\int_{\mathbb{R}^{d}}|f(t)|^{p} m(t)^{p} d t\right)^{1 / p} .
$$

If $p=\infty$, then $f \in L_{m}^{\infty}$ means that $|f(t)| \leq\|f\|_{L_{m}^{\infty}} m(t)^{-1}$. Likewise $\ell_{m}^{p}\left(\mathbb{Z}^{d}\right)$ is defined by the norm $\|\mathbf{a}\|_{\ell_{m}^{p}}=\|\mathbf{a} m\|_{\ell^{p}}$.

The moderateness of a weight is exactly the condition required for convolution estimates in the style of Young's inequality [11].

Lemma 5.1. Assume that $v$ is submultiplicative on $\mathbb{Z}^{d}$. Then the following are equivalent:

(i) $m$ is v-moderate.

(ii) $\ell_{m}^{p}\left(\mathbb{Z}^{d}\right)$ is invariant under the shift $T_{k}$ and the operator norm satisfies

$$
\left\|T_{k}\right\|_{\ell_{m}^{p} \rightarrow \ell_{m}^{p}} \leq C v(k) \quad \text { for } k \in \mathbb{Z}^{d} .
$$

(iii) The convolution relation $\ell_{v}^{1} * \ell_{m}^{p} \subseteq \ell_{m}^{p}$ holds in the sense of the norm estimate

$$
\|\mathbf{a} * \mathbf{b}\|_{\ell_{m}^{p}} \leq C\|\mathbf{a}\|_{\ell_{v}^{1}}\|\mathbf{b}\|_{\ell_{m}^{p}} .
$$

Similarly, on $\mathbb{R}^{d}$ the convolution relation $L_{v}^{1} * L_{m}^{p} \subseteq L_{m}^{p}$ holds if and only if $m \in \mathcal{M}_{v}$. Proof. The argument is similar to Lemma 4.1, See [11] for a detailed proof.

Whereas $L_{v}^{1}$ is a Banach algebra, $L_{m}^{p}$ is only a Banach space in general. Lemma 5.1 states that $L_{v}^{1}$ acts continuously on $L_{m}^{p}$ by convolution. In algebraic terminology, $L_{m}^{p}$ is an $L_{v}^{1}$-convolution-module. The lemma is the prototype and explains why and where moderate weights occur. 
5.1. Modulation Spaces. In time-frequency analysis moderate weights occur in the definition of general modulation spaces. These spaces are defined in terms of a function space norm applied to the short-time Fourier transform $V_{g} f$. The idea is to measure the time-frequency concentration of a function or distribution. There are several equivalent definitions. We use the most general definition as discussed in the general theory of coorbit spaces [12].

Definition 5. Assume that $v$ is submultiplicative and choose $g \in M_{v}^{1}, g \neq 0$. Let $\left(M_{v}^{1}\right)^{\sim}$ be the space of all conjugate linear functionals on $M_{v}^{1}$. Let $m \in \mathcal{M}_{v}$ and $1 \leq p, q \leq \infty$. Then the modulation space $M_{m}^{p, q}\left(\mathbb{R}^{d}\right)$ consists of all $f \in\left(M_{v}^{1}\right)^{\sim}\left(\mathbb{R}^{d}\right)$, such that $V_{g} f \in L_{m}^{p, q}\left(\mathbb{R}^{2 d}\right)$, and the norm is

$$
\|f\|_{M_{m}^{p, q}}=\left\|V_{g} f\right\|_{L_{m}^{p, q}}=\left(\int_{\mathbb{R}^{d}}\left(\int_{\mathbb{R}^{d}}\left|V_{g} f(x, \xi)\right|^{p} m(x, \xi)^{p} d x\right)^{q / p} d \xi\right)^{1 / q} .
$$

If $p=\infty$ or $q=\infty$, then we use the supremum norm.

We now state the main properties; these are essential for a meaningful theory of these function and distribution spaces [15, Ch. 11].

Theorem 5.2. Let $m \in \mathcal{M}_{v}, 1 \leq p, q \leq \infty$.

(a) Then the modulation space $M_{m}^{p, q}\left(\mathbb{R}^{d}\right)$ is a Banach space.

(b) If $h \in M_{v}^{1}, h \neq 0$, then

$$
\left\|V_{h} f\right\|_{L_{m}^{p, q}} \asymp\left\|V_{g} f\right\|_{L_{m}^{p, q}} .
$$

Thus the definition of $M_{m}^{p, q}$ is independent of the window $g$, and different window in $M_{v}^{1}$ yield equivalent norms on $M_{m}^{p, q}$.

(c) Invariance under time-frequency shifts: If $X=(x, \xi) \in \mathbb{R}^{2 d}$, then

$$
\|\pi(X) f\|_{M_{m}^{p, q}} \leq C v(X)\|f\|_{M_{m}^{p, q}} .
$$

Let us briefly sketch why the weight $m$ in Definition 5 and Theorem 5.2 has to be moderate. We use the covariance property of the STFT (11) in the form ||$V_{g}(\pi(X) f)(Y)|=| V_{g} f(Y-X)\left|=T_{X}\right| V_{g} f \mid(Y)$. Consequently, if $m$ is $v$-moderate, then by Lemma 5.1

$$
\|\pi(X) f\|_{M_{m}^{p, p}}=\left\|V_{g}(\pi(X) f)\right\|_{L_{m}^{p}}=\left\|T_{X} V_{g} f\right\|_{L_{m}^{p}} \leq C v(X)\|f\|_{M_{m}^{p, p}} .
$$

Thus the translation invariance of $L_{m}^{p}$ implies the invariance of $M_{m}^{p, p}$ under timefrequency shifts.

The norm equivalence (24) is based on a fundamental pointwise inequaliy for STFTs [15, Lemma 11.3.2]

$$
\left|V_{h} f(X)\right| \leq\left(\left|V_{g} f\right| *\left|V_{h} g\right|\right)(X) \quad X \in \mathbb{R}^{2 d} .
$$

If $V_{g} f \in L_{m}^{p}$ and $V_{h} g \in L_{v}^{1}$, then $V_{h} f \in L_{m}^{p}$ by Lemma 5.1, and once again $m$ needs to be $v$-moderate.

For a meaningful definition of the modulation spaces we need (a) a reasonable space of test functions that is invariant under time-frequency shifts, and (b) a corresponding space of distributions. Then the STFT is well defined and $M_{m}^{p, q}$ consists of all distributions such that $V_{g} f \in L_{m}^{p, q}$. Definition 5 is formulated with the spaces 
of test functions and distributions that are intrinsic to time-frequency analysis. Specifically, the appropriate spaces of test functions in time-frequency analysis are the spaces of "good windows" $M_{v}^{1}$, and the appropriate spaces of distributions are the dual spaces $\left(M_{v}^{1}\right)^{\tilde{}}$. This approach works even for the exponential weights $v(X)=e^{a|X|}$.

For reasons of convenience, many authors have treated modulation spaces for a more restrictive class of weight functions, so that standard concepts can be used.

Proposition 5.3. If $v$ grows at most polynomially, i.e., $v(X) \leq C(1+|X|)^{N}, X \in$ $\mathbb{R}^{2 d}$ for some constants $C, N \geq 0$, and $m \in \mathcal{M}_{v}$, then $M_{m}^{p, q}$ is a subspace of the tempered distributions, and so

$$
M_{m}^{p, q}=\left\{f \in \mathcal{S}^{\prime}\left(\mathbb{R}^{d}\right): V_{g} f \in L_{m}^{p, q}\right\},
$$

whenever $g \in \mathcal{S}, g \neq 0$.

Several alternative spaces of test functions have been proposed after the coorbit space approach in [12]:

1. Let $\varphi(t)=e^{-\pi t^{2}}$ be the Gaussian on $\mathbb{R}^{d}$, then

$$
\mathcal{S}_{\mathcal{C}}=\left\{g \in L^{2}\left(\mathbb{R}^{d}\right): g=\int_{\mathbb{R}^{2 d}} F(X) \pi(X) \varphi d X, \operatorname{supp} F \text { is compact }\right\}
$$

is a suitable space of test functions that works for all exponential weights $[15$, Chpt. 11.4].

2. In a similar spirit, a discrete version of (28), namely

$$
\mathcal{S}_{\mathcal{C}, F}=\left\{g: g=\sum_{j=1}^{n} c_{j} \pi\left(X_{j}\right) \varphi \text { for } c_{j} \in \mathbb{C}, X_{j} \in \mathbb{R}^{2 d}\right\},
$$

was proposed in [7] as a suitable window class. Then $M_{m}^{p, q}$ can also be defined as the norm completion of $\mathcal{S}_{\mathcal{C}, F}$ with respect to the $M_{m}^{p, q}$-norm.

3. Another choice is the Gelfand-Shilov space $S^{1 / 2,1 / 2}$, which is used in [8].

For any submultiplicative weight $v$, we have the embeddings $\mathcal{S}_{\mathcal{C}, F} \subseteq S^{1 / 2,1 / 2} \subseteq$ $M_{v}^{1}$ and $\mathcal{S}_{\mathcal{C}} \subseteq S^{1 / 2,1 / 2} \subseteq M_{v}^{1}$, and by [15, Prop. 11.4.2] each of $\mathcal{S}_{\mathcal{C}}, \mathcal{S}_{\mathcal{C}, F}$ and $S^{1 / 2,1 / 2}$ is dense in $M_{v}^{1}$. Consequently, these spaces of "special windows" are universal and work for an arbitrary submultiplicative weight function $v$.

Beside the natural distribution spaces $M_{1 / v}^{\infty}=\left(M_{v}^{1}\right)^{\sim}$ several other spaces have been studied recently. In some applications $m$ is allowed to grow faster than polynomially, then it is necessary to leave the realm of the Schwartz class and tempered distributions. If $m(X)=\mathcal{O}\left(e^{a|X|^{b}}\right), 0 \leq b<1$, then the correct distribution space is the space of ultradistributions of Björck [5] and Komatsu [30]. For this reason, the $M_{m}^{p, q}$ were renamed ultra-modulation spaces by Pilipovic and Teofanov [36]. If $m$ grows exponentially, e.g., $m(X)=e^{a|X|}$, then it can be shown that $M_{m}^{p, q}$ is contained in the Gelfand-Shilov space $\left(S^{1 / 2,1 / 2}\right)^{\prime}[8]$. Or to say it differently, all modulation spaces with a moderate weight function are contained in $\left(S^{1 / 2,1 / 2}\right)^{\prime}$.

With an appropriate distribution space at hand, modulation spaces can be defined for arbitrary moderate weight functions $m$. In particular no restriction needs to be imposed on the growth of the moderate weight $m$. 


\subsection{Twisted Convolution of Weighted $\ell^{p}$-Spaces.}

Proposition 5.4. If $m$ is $v$-moderate, then $\ell_{v}^{1} \sharp \ell_{m}^{p} \subseteq \ell_{m}^{p}$ with the norm estimate

$$
\|\mathbf{a} \sharp \mathbf{b}\|_{\ell_{m}^{p}} \leq C\|\mathbf{a}\|_{\ell_{v}^{1}}\|\mathbf{b}\|_{\ell_{m}^{p}} .
$$

Proof. Since $|(\mathbf{a} \sharp \mathbf{b})(k, l)| \leq(|\mathbf{a}| *|\mathbf{b}|)(k, l)$, (29) follows from Theorem 5.1 (Young's inequality).

5.3. Twisted Product between Modulation Spaces. A "module property" of modulation spaces with respect to the twisted product can be formulated as follows.

Theorem 5.5. If $v$ is submultiplicative on $\mathbb{R}^{2 d}$ and $m \in \mathcal{M}_{v}$, then $M_{m}^{\infty, p}$ is an $M_{v}^{\infty, 1}$-module with respect to $\circ$. This means that the Young-type inequality

$$
\|\sigma \circ \tau\|_{M_{m}^{\infty, p}} \leq C\|\sigma\|_{M_{v}^{\infty, 1}}\|\tau\|_{M_{m}^{\infty, p}}
$$

holds for all $\sigma \in M_{v}^{1}$ and all $\tau \in M_{m}^{\infty, p}$.

Proof. The proof follows from estimate (26) for the grand symbols and Young's Theorem 5.1.

\section{GRS-WEIGHTS} if

A submultiplicative weight $v$ satisfies the Gelfand-Raikov-Shilov condition (GRS),

$$
\lim _{n \rightarrow \infty} v(n x)^{1 / n}=1 \quad \forall x \in \mathbb{R}^{d} .
$$

The subexponential weight $e^{a|x|^{b}}$ for $a>0$ and $0 \leq b<1$ satisfies the GRScondition, but the exponential weight $e^{a|x|}$ violates the GRS-condition. Intuitively, the GRS-condition describes the subexponential growth of a weight in precise technical terms and excludes all forms of exponential growth.

$$
\text { GRS-weights characterize spectral invariance. }
$$

6.1. Wiener's Lemma. To prepare the background for the GRS-condition, we first recall the original version of Wiener's lemma for absolutely convergent Fourier series.

Theorem 6.1. Assume that $f$ is an absolutely converging Fourier series such that $f(t) \neq 0$ for all $t \in \mathbb{T}$, then $1 / f$ is also an absolutely convergent Fourier series.

For weighted versions of Wiener's Lemma, we define

$$
\mathcal{A}_{v}\left(\mathbb{T}^{d}\right)=\left\{f: f(t)=\sum_{k \in \mathbb{Z}^{d}} a_{k} e^{2 \pi i k \cdot t}, \mathbf{a}=\left(a_{k}\right) \in \ell_{v}^{1}\left(\mathbb{Z}^{d}\right)\right\}
$$

to be the space of weighted absolutely convergent Fourier series. We equip $\mathcal{A}_{v}\left(\mathbb{T}^{d}\right)$ with the norm $\|f\|_{\mathcal{A}_{v}}=\|\mathbf{a}\|_{\ell_{v}^{1}}$, then $\mathcal{A}_{v}$ is a Banach algebra with respect to pointwise multiplication. 
The weighted version of Wiener's Lemma requires the GRS-condition and is taken from [14].

Theorem 6.2. Assume that $v$ is a submultiplicative weight on $\mathbb{Z}^{d}$ satisfying the $G R S$-condition. If $f \in \mathcal{A}_{v}\left(\mathbb{T}^{d}\right)$ and $f(t) \neq 0$ for all $t \in \mathbb{T}^{d}$, then $1 / f$ is also in $\mathcal{A}_{v}\left(\mathbb{T}^{d}\right)$.

6.2. Inverse-Closedness. Though not immediate, Wiener's Lemma should be understood as a statement about the relation between two Banach algebras. We first define the abstract concept.

Definition 6. Let $\mathcal{A} \subseteq \mathcal{B}$ be two Banach algebras with a common identity $e$. We say that $\mathcal{A}$ is inverse-closed in $\mathcal{B}$, if

$$
a \in \mathcal{A} \text { and } a^{-1} \in \mathcal{B} \Rightarrow a^{-1} \in \mathcal{A} \text {. }
$$

In other words, "the invertibility in the big algebra implies the invertibility in the small algebra."

In the case of Wiener's Lemma we take $\mathcal{A}=\mathcal{A}_{v}\left(\mathbb{T}^{d}\right)$ and $\mathcal{B}=C\left(\mathbb{T}^{d}\right)$ or $\mathcal{B}=$ $L^{\infty}\left(\mathbb{T}^{d}\right)$ (all with pointwise multiplication). If $f \in \mathcal{A}_{v}$ does not vanish anywhere, then by continuity $\inf _{t \in \mathbb{T}^{d}}|f(t)|>0$ and $f$ is invertible in $C\left(\mathbb{T}^{d}\right)$ (or in $L^{\infty}\left(\mathbb{T}^{d}\right)$ ). Wiener's Lemma says that $1 / f$ must already be in the small algebra $\mathcal{A}_{v}\left(\mathbb{T}^{d}\right)$ of weighted absolutely convergent Fourier series. So Wiener's Lemma can be recast by saying that $\mathcal{A}_{v}\left(\mathbb{T}^{d}\right)$ is inverse-closed in $C\left(\mathbb{T}^{d}\right)$.

Naimark [34] turned Wiener's Lemma into a definition and calls a nested pair of Banach algebras with common identity a Wiener pair, if $\mathcal{A}$ is inverse-closed in $\mathcal{B}$.

Inverse-closedness can be understood as a spectral property. Let $\sigma_{\mathcal{A}}(a)=\{\lambda \in$ $\mathbb{C}: a-\lambda e$ is not invertible in $\mathcal{A}\}$ denote the spectrum of an element $a \in \mathcal{A}$. If $\mathcal{A}=C\left(\mathbb{T}^{d}\right)$, then $\sigma_{C\left(\mathbb{T}^{d}\right)}(f)=\operatorname{ran} f=f\left(\mathbb{T}^{d}\right)$.

The following statement is a simple reformulation of Definition 6 .

Lemma 6.3 (Spectral invariance, spectral permanence). Let $\mathcal{A} \subseteq \mathcal{B}$ be two Banach algebras with a common identity e. Then $\mathcal{A}$ is inverse-closed in $\mathcal{B}$, if and only if

$$
\sigma_{\mathcal{A}}(a)=\sigma_{\mathcal{B}}(a) \quad \forall a \in \mathcal{A}
$$

We now have enough abstract background to reformulate Wiener's Lemma and understand the full meaning of the GRS-condition.

Theorem 6.4. The spectral identity $\sigma_{\mathcal{A}_{v}\left(\mathbb{T}^{d}\right)}(f)=\sigma_{C\left(\mathbb{T}^{d}\right)}(f)=\operatorname{ran} f$ holds for all $f \in \mathcal{A}_{v}\left(\mathbb{T}^{d}\right)$ if and only if $v$ satisfies the GRS-condition.

Counter-Example. Whereas the sufficiency of the GRS-condition is quite subtle and is based on Gelfand theory for commutative Banach algebras and complex analysis, the necessity is easy to understand by a counter-example. The following reveals the essence of the GRS-condition.

Assume that $v$ violates the GRS-condition. Then there exists a $k \in \mathbb{Z}^{d}$ such that $\lim _{n \rightarrow \infty} v(n k)^{1 / n}=a=e^{\alpha}>1$, and so $v(n k) \geq e^{\alpha n / 2}$ for $n \geq n_{0}$. Thus $v$ grows exponentially in some direction. 
Let $0<\delta<\alpha / 2$ and set $f(t)=1-e^{-\delta} e^{2 \pi i k \cdot t}$. Since $f$ is a trigonometric polynomial, we have $f \in \mathcal{A}_{v}\left(\mathbb{T}^{d}\right)$ and clearly $|f(t)| \geq 1-e^{-\delta}>0$ for all $t$. The inverse of $f$ is the geometric series

$$
\frac{1}{f(t)}=\frac{1}{1-e^{-\delta} e^{2 \pi i k \cdot t}}=\sum_{n=0}^{\infty} e^{-n \delta} e^{2 \pi i n k \cdot t} .
$$

So $1 / f$ is an absolutely convergent Fourier series, but

$$
\|1 / f\|_{\mathcal{A}_{v}}=\sum_{n=0}^{\infty} e^{-n \delta} v(n k) \geq \sum_{n=n_{0}}^{\infty} e^{-n \delta} e^{n \alpha / 2}=\infty,
$$

and so $1 / f \notin \mathcal{A}_{v}\left(\mathbb{T}^{d}\right)$.

6.3. Convolution Operators on $L_{v}^{1}$. Wiener's Lemma can be interpreted as a statement about convolution operators. Let $C_{\mathbf{a}}$ be the convolution operator defined by $C_{\mathbf{a}} \mathbf{c}=\mathbf{a} * \mathbf{c}$ for $\mathbf{a} \in \ell^{1}\left(\mathbb{Z}^{d}\right)$ and $\mathbf{c} \in \ell^{2}\left(\mathbb{Z}^{d}\right)$. We identify $\ell_{v}^{1}\left(\mathbb{Z}^{d}\right)$ with the subalgebra Op $\left(\ell_{v}^{1}\right):=\left\{C_{\mathbf{a}}: \mathbf{a} \in \ell_{v}^{1}\left(\mathbb{Z}^{d}\right)\right\}$ of the $\mathrm{C}^{*}$-algebra $\mathcal{B}\left(\ell^{2}\left(\mathbb{Z}^{d}\right)\right)$ of all bounded operators on $\ell^{2}\left(\mathbb{Z}^{d}\right)$. Then Theorem 6.4 is equivalent to the following.

Theorem 6.5. The spectral invariance $\sigma_{\ell_{v}^{1}}(\mathbf{a})=\sigma_{\mathcal{B}\left(\ell^{2}\right)}\left(C_{\mathbf{a}}\right)=\operatorname{ran} \widehat{\mathbf{a}}$ holds for all $\mathbf{a} \in \ell_{v}^{1}\left(\mathbb{Z}^{d}\right)$, if and only if $v$ satisfies the $G R S$-condition.

To see how this statement follows from Theorem 6.4, we take Fourier series $\widehat{\mathbf{a}}(\xi)=\sum_{k \in \mathbb{Z}^{d}} a_{k} e^{2 \pi i k \cdot \xi}$. Then $\left(C_{\mathbf{a}} \mathbf{c}\right)^{\widehat{ }}=\widehat{\mathbf{a}} \widehat{\mathbf{c}}$ and $C_{\mathbf{a}}$ is unitarily equivalent to the multiplication operator by $\widehat{\mathbf{a}}$, which has the spectrum ran $\widehat{\mathbf{a}}$.

Replacing the group $\mathbb{Z}^{d}$ by some possibly non-commutative locally compact group $G$, we may ask whether and for which groups a version of Theorem 6.4 still holds. The most general result we know of characterizes again GRS-weights.

Theorem 6.6 ( [13]). Let $G$ be a compactly generated group of polynomial growth and $v$ a submultiplicative weight on $G$. Then the spectral identity $\sigma_{L_{v}^{1}(G)}(f)=$ $\sigma_{\mathcal{B}\left(L^{2}\right)}\left(C_{f}\right)$ holds for all $f \in L_{v}^{1}(G)$, if and only if $v$ satisfies the $G R S$-condition on $G$, which is $\lim _{n \rightarrow \infty} v\left(x^{n}\right)^{1 / n}=1$ for all $x \in G$.

6.4. Wiener's Lemma for Twisted Convolution. The version of Wiener's Lemma in the form of Theorem 6.5 suggests that we study the spectrum of the twisted convolution operator $C_{\mathbf{a}} \mathbf{c}=\mathbf{a} \sharp \mathbf{c}$. Although Fourier series are no longer available for the non-commutative convolution $\sharp$, Wiener's Lemma holds also for twisted convolution. Again, we identify the algebra $\ell_{v}^{1}\left(\mathbb{Z}^{2 d}\right)$ with a subalgebra of $\mathcal{B}\left(\ell^{2}\right)$ via the isomorphism $\mathbf{a} \rightarrow C_{\mathbf{a}}$.

Theorem 6.7. The algebra $\ell_{v}^{1}\left(\mathbb{Z}^{2 d}\right)$ is inverse-closed in $\mathcal{B}\left(\ell^{2}\left(\mathbb{Z}^{2 d}\right)\right)$, if and only if $v$ satisfies the $G R S$-condition. In particular, if $\mathbf{a} \in \ell_{v}^{1}\left(\mathbb{Z}^{2 d}\right)$ and if the operator $\mathbf{c} \rightarrow C_{\mathbf{a}} \mathbf{c}=\mathbf{a} \sharp \mathbf{c}$ is invertible on $\ell^{2}\left(\mathbb{Z}^{2 d}\right)$, then there is a $\mathbf{b} \in \ell_{v}^{1}\left(\mathbb{Z}^{2 d}\right)$, such that $\mathbf{a} \sharp \mathbf{b}=\delta$ and $C_{\mathbf{a}}^{-1}=C_{\mathbf{b}}$. 
The proof is highly non-trivial. In [19] only radial weight functions were considered; the general case can be deduced from the general Theorem 6.6. We note that several other proofs have been found in the meantime, see [3,20].

Since $\ell_{v}^{1}\left(\mathbb{Z}^{2 d}\right)$ and the algebra of weigthed absolutely convergent series of timefrequency shifts $\mathcal{A}_{v}(\alpha, \beta)$ are isomorphic, we obtain Wiener's Lemma for the rotation algebra.

Corollary 6.8. The rotation algebra $\mathcal{A}_{v}(\alpha, \beta)$ is inverse-closed in $\mathcal{B}\left(L^{2}\left(\mathbb{R}^{d}\right)\right)$, if and only if $v$ satisfies the GRS-condition. Consequently, if $A \in \mathcal{A}_{v}(\alpha, \beta)$ and $A$ is invertible on $L^{2}\left(\mathbb{R}^{d}\right)$, then $A^{-1} \in \mathcal{A}_{v}(\alpha, \beta)$.

Although Corollary 6.8 seems to be an innocent result in time-frequency analysis, it plays an important role in operator algebras and non-commutative geometry, and occurs in the work of Connes [6], Rieffel [38], and Arveson [1]. The precise connections have been discovered by F. Luef [32]. Another version of Wiener's Lemma for absolutely convergent series of time-frequency shifts have been given recently by Balan [2].

6.5. Gabor frames. Next we look at the theory of Gabor frames and their duals. Let $\Lambda \subseteq \mathbb{R}^{2 d}$ be a lattice in the time-frequency plane. Every lattice is of the form $\Lambda=A \mathbb{Z}^{2 d}$, where $A$ is some invertible $2 d \times 2 d$-matrix. Given a function $g \in L^{2}(\mathbb{R})$, we write $\mathcal{G}(g, \Lambda)$ for the set $\{\pi(\lambda) g: \lambda \in \Lambda\}$. The set $\mathcal{G}(g, \Lambda)$ is called a Gabor frame for $L^{2}\left(\mathbb{R}^{d}\right)$, if the associated frame operator

$$
S f=S_{g, \Lambda} f=\sum_{\lambda \in \Lambda}\langle f, \pi(\lambda) g\rangle \pi(\lambda) g
$$

is invertible on $L^{2}$. Equivalently, there exist constants $A, B>0$, such that

$$
A\|f\|_{2}^{2} \leq \sum_{\lambda \in \Lambda}|\langle f, \pi(\lambda) g\rangle|^{2} \leq B\|f\|_{2}^{2} \quad \forall f \in L^{2}\left(\mathbb{R}^{d}\right) .
$$

We note that $S$ commutes with all time-frequency shifts $\pi(\lambda)$ for $\lambda \in \Lambda$ and by the spectral theorem $f(S)$ also commutes with all $\pi(\lambda)$. If $S$ is invertible, then in particular $S^{-1}(\pi(\lambda) g)=\pi(\lambda) S^{-1} g$ and also $S^{-1 / 2}(\pi(\lambda) g)=\pi(\lambda) S^{-1 / 2} g$ for all $\lambda \in \Lambda$. The window $\gamma=S^{-1} g$ is called the canonical dual window, and $\gamma^{\circ}=S^{-1 / 2} g$ is the canonical tight window associated to $g$.

If $\mathcal{G}(g, \Lambda)$ is a Gabor frame, then every $f \in L^{2}\left(\mathbb{R}^{d}\right)$ can be reconstructed from the frame coefficients $\langle f, \pi(\lambda) g\rangle$ by the formula

$$
\begin{aligned}
f & =S^{-1} S f=\sum_{\lambda \in \Lambda}\langle f, \pi(\lambda) g\rangle S^{-1} \pi(\lambda) g \\
& =\sum_{\lambda \in \Lambda}\langle f, \pi(\lambda) g\rangle \pi(\lambda) \gamma
\end{aligned}
$$

The factorizations $\mathrm{I}=S S^{-1}$ and $\mathrm{I}=S^{-1 / 2} S S^{-1 / 2}$ lead to similar expansion formulas. The former factorization yields the expansion with respect to the Gabor frame 
$\mathcal{G}(g, \Lambda)$

$$
\begin{aligned}
f & =S S^{-1} f=\sum_{\lambda \in \Lambda}\left\langle f, S^{-1} \pi(\lambda) g\right\rangle \pi(\lambda) g \\
& =\sum_{\lambda \in \Lambda}\langle f, \pi(\lambda) \gamma\rangle \pi(\lambda) g
\end{aligned}
$$

and the latter factorization yields the so-called tight frame expansion

$$
\begin{aligned}
f & =S^{-1 / 2} S S^{-1 / 2} f=\sum_{\lambda \in \Lambda}\left\langle S^{-1 / 2} f, \pi(\lambda) g\right\rangle S^{-1 / 2} \pi(\lambda) g \\
& =\sum_{\lambda \in \Lambda}\left\langle f, \pi(\lambda) \gamma^{\circ}\right\rangle \pi(\lambda) \gamma^{\circ} .
\end{aligned}
$$

All three expansions are pure Hilbert space theory and are based solely on the invertibility of the Gabor frame operator $S$ on $L^{2}\left(\mathbb{R}^{d}\right)$. For genuine time-frequency analysis, the series expansions are required to converge in other norms than $L^{2}$. The smoothness and the decay of a function or distribution should be encoded in the frame coefficients $\langle f, \pi(\lambda) g\rangle$. For this purpose, we need to impose additional conditions on the window $g$.

The key lies in the qualities of the dual window and of the tight dual window. The main theorem in this regard states that all three windows $g, \gamma$, and $\gamma^{\circ}$ possess the same time-frequency localization.

Theorem 6.9. Assume that $v$ is submultiplicative on $\mathbb{R}^{2 d}$ and satisfies the GRScondition. If $\mathcal{G}(g, \Lambda)$ is a Gabor frame for $L^{2}\left(\mathbb{R}^{d}\right)$ and $g \in M_{v}^{1}$, then $\gamma=S^{-1} g$ and $\gamma^{\circ}=S^{-1 / 2} g$ are also in $M_{v}^{1}$.

The proof can be based on a version of Corollary 6.8 for general time-frequency lattices $\Lambda$, but for simplicity we assume that $\Lambda=\alpha \mathbb{Z}^{d} \times \beta \mathbb{Z}^{d}$. By a result of G. Janssen [29] the Gabor frame operator $S_{g, \Lambda}$ can be represented as a series of certain time-frequency shifts. Precisely, if $g \in M_{v}^{1}$, then $S \in \mathcal{A}_{v}\left(\beta^{-1}, \alpha^{-1}\right)$. Now Corollary 6.8 implies that $S^{-1} \in \mathcal{A}_{v}\left(\beta^{-1}, \alpha^{-1}\right)$. One concludes by showing that $A \in \mathcal{A}_{v}\left(\beta^{-1}, \alpha^{-1}\right)$ and $g \in M_{v}^{1}$ always imply that $A g \in M_{v}^{1}$. By stretching the arguments slightly, one arrives at the following reformulation taken from [12].

Theorem 6.10. Assume that $g \in M_{v}^{1}$ for some submultiplicative weight satisfying the GRS-condition and that $\Lambda=\alpha \mathbb{Z}^{d} \times \beta \mathbb{Z}^{d}$. Then the following are equivalent:

(i) The frame operator $S_{g, \Lambda}$ is invertible on $L^{2}\left(\mathbb{R}^{d}\right)$.

(ii) $S$ is invertible on $M_{v}^{1}$.

(iii) There exist indices $p, q \in[1, \infty]$ and a moderate weight function $m \in \mathcal{M}_{v}$, such that $S$ is invertible on the modulation space $M_{m}^{p, q}$.

(iv) $S$ is invertible on all modulation spaces $M_{m}^{p, q}$ for all $p, q \in[1, \infty]$ and all $m \in \mathcal{M}_{v}$.

Using the well-developed machinery of modulation space techniques, we can prove the following version of time-frequency analysis for distributions. 
Theorem 6.11. Assume that $g \in M_{v}^{1}$ for some $v$ submultiplicative weight satisfying the GRS-condition and that $\mathcal{G}(g, \Lambda)$ is a Gabor frame for $L^{2}\left(\mathbb{R}^{d}\right)$. Then the following properties hold for all $v$-moderate weights $m \in \mathcal{M}_{v}$ :

(a) If $f \in M_{m}^{p, q}$, then the frame expansions in (35) - (37) converge in the norm of $M_{m}^{p, q}$ for $1 \leq p, q<\infty$ and weak-* when $p q=\infty$.

(b) Norm equivalence:

$$
\begin{aligned}
&\|f\|_{M_{m}^{p, p}} \asymp\left\|\langle f, \pi(\lambda) g\rangle_{\lambda \in \Lambda}\right\|_{\ell_{m}^{p}} \\
& \asymp\left\|\langle f, \pi(\lambda) \gamma\rangle_{\lambda \in \Lambda}\right\|_{\ell_{m}^{p}} \\
& \asymp\left\|\left\langle f, \pi(\lambda) \gamma^{0}\right\rangle_{\lambda \in \Lambda}\right\|_{\ell_{m}^{p}}
\end{aligned}
$$

(c) If $\Lambda=\alpha \mathbb{Z}^{d} \times \beta \mathbb{Z}^{d}$, then we also have

$$
\|f\|_{M_{m}^{p, q}} \asymp\left\|\langle f, \pi(\lambda) g\rangle_{\lambda \in \Lambda}\right\|_{\ell_{m}^{p, q}} \asymp\left\|\langle f, \pi(\lambda) \gamma\rangle_{\lambda \in \Lambda}\right\|_{\ell_{m}^{p}} \asymp\left\|\left\langle f, \pi(\lambda) \gamma^{\circ}\right\rangle_{\lambda \in \Lambda}\right\|_{\ell_{m}^{p}} .
$$

Expressed in technical jargon, Theorem 6.11 says that a Gabor frame $\mathcal{G}\left(g, \alpha \mathbb{Z}^{d} \times\right.$ $\beta \mathbb{Z}^{d}$ ) with $g \in M_{v}^{1}$ is a Banach frame for the entire family of modulation spaces $M_{m}^{p, q}$. Once again, the class of admissible weights $m$ consists exactly of the $v$ moderate weights, where $v$ parametrizes the time-frequency concentration of the window $g$. For detailed proofs see [12] and [15, Ch. 12].

6.6. Universal Gabor Frames. The above results exclude the use of exponential weights such as $m(z)=e^{a|z|}$ for $a \in \mathbb{R}$, and they do not guarantee a decent timefrequency analysis for a modulation space $M_{m}^{p, q}$ with exponential weight $m$.

For exponential weights the Banach algebra methods used in the proofs of the spectral invariance property do fail. We have to resort to different methods. The following theorem is implicit in the work of Seip, Lyubarski [33,39], and also of Janssen [28].

Theorem 6.12. Let $\varphi(t)=e^{-\pi t^{2}}, t \in \mathbb{R}^{d}$ and $\alpha \beta<1$. Then $\mathcal{G}\left(\varphi, \alpha \mathbb{Z}^{d} \times \beta \mathbb{Z}^{d}\right)$ is a frame for $L^{2}\left(\mathbb{R}^{d}\right)$. Moreover, there exists a dual window $\gamma$ (not necessarily the canonical dual window $S^{-1} \mathrm{~g}$ ) such that

$$
\left|V_{\varphi} \gamma(z)\right| \leq C e^{(\alpha \beta-1) \pi|z|^{2} / 2} \quad z \in \mathbb{R}^{2 d} .
$$

Consequently $\gamma$ belongs to $\bigcap_{v} M_{v}^{1}$, where the intersection is over all submultiplicative weights.

The proof of this theorem is quite ingenious: By means of the Bargmann transform the frame property of $\mathcal{G}(\varphi, \Lambda)$ is translated into an equivalent problem of sampling and interpolation in the Bargmann-Fock space. The solution to this problem is provided by a modification of the Weierstraß sigma function associated to the lattice $\Lambda$. The crucial decay estimate (39) then follows from a subtle and explicit growth estimate of that sigma function. See the original literature for details and [21] for a simple approach that also works for the class of Hermite functions.

As a consequence, the time-frequency analysis of modulation spaces of Theorem 6.11 works for all modulation spaces $M_{m}^{p, q}$ without any restriction on the weight $m$ provided that is is moderate. 
Corollary 6.13. Assume that $\alpha \beta<1$ and let $\gamma$ be the dual window of $\varphi$ guaranteed by Theorem 6.12. Then the following properties hold for an arbitrary moderate weight function $m$, even when $m$ grows exponentially.

(a) If $f \in M_{m}^{p, q}$, then the frame expansions (35) and (36) converge in the norm of $M_{m}^{p, q}$ for $1 \leq p, q<\infty$ and weak-* for $p q=\infty$.

(b) Norm equivalence: For all $f \in M_{m}^{p, q}$ we have

$$
\|f\|_{M_{m}^{p, q}} \asymp\left\|\langle f, \pi(\lambda) g\rangle_{\lambda \in \Lambda}\right\|_{\ell_{m}^{p, q}}
$$

6.7. The Wiener Property of Sjöstrand's Class $M_{v}^{\infty, 1}$. We have seen that the modulation space $M_{v}^{\infty, 1}\left(\mathbb{R}^{2 d}\right)$ is a Banach algebra with respect to the product $\circ$ that corresponds to the composition of two pseudodifferential operators. In analogy to convolution operators, we identify symbols with the corresponding pseudodifferential operators by defining

$$
\mathrm{Op}\left(M_{v}^{\infty, 1}\right)=\left\{K_{\sigma}: \sigma \in M_{v}^{\infty, 1}\right\} .
$$

Then $\mathrm{Op}\left(M_{v}^{\infty, 1}\right)$ is a subalgebra of $\mathcal{B}\left(L^{2}\left(\mathbb{R}^{d}\right)\right)$. Sjöstrand [41] proved the fundamental result that $\mathrm{Op}\left(M^{\infty, 1}\right)$ is inverse-closed in $\mathcal{B}\left(L^{2}\left(\mathbb{R}^{d}\right)\right)$.

Theorem 6.14. If $\sigma \in M^{\infty, 1}$ and the corresponding pseudodifferential operator $K_{\sigma}$ is invertible on $L^{2}\left(\mathbb{R}^{d}\right)$, then there exists a symbol $\tau \in M^{\infty, 1}$, such that $K_{\tau}=K_{\sigma}^{-1}$, in other words, the algebra $\mathrm{Op}\left(M^{\infty, 1}\right)$ is inverse-closed in $\mathcal{B}\left(L^{2}\left(\mathbb{R}^{d}\right)\right)$.

The weighted version was obtained in [17]. It is not surprising that the GRScondition occurs once more.

Theorem 6.15. Assume that $v$ is a submultiplicative weight on $\mathbb{R}^{2 d}$ satisfying the GRS-condition. If $\sigma \in M_{v}^{\infty, 1}$ and $K_{\sigma}$ is invertible on $L^{2}\left(\mathbb{R}^{d}\right)$, then $K_{\sigma}^{-1}=K_{\tau}$ for some $\tau \in M_{v}^{\infty, 1}$. Thus $\mathrm{Op}\left(M_{v}^{\infty, 1}\right)$ is inverse-closed in $\mathcal{B}\left(L^{2}\left(\mathbb{R}^{d}\right)\right)$ or equivalently,

$$
\sigma_{M_{v}^{\infty, 1}}(\tau)=\sigma_{\mathcal{B}\left(L^{2}\right)}\left(K_{\tau}\right) \quad \forall \tau \in M_{v}^{\infty, 1} .
$$

To highlight the role of the GRS-condition, we reformulate Theorem 6.15 as follows.

Corollary 6.16. The spectral invariance $\sigma_{M_{v}^{\infty, 1}}(\tau)=\sigma_{\mathcal{B}\left(L^{2}\right)}\left(K_{\tau}\right)$ holds for all $\tau \in$ $M_{v}^{\infty, 1}$ if and only if $v$ satisfies the GRS-condition.

The necessity of the GRS-condition is verified as in the proof of Theorem6.4. If $v$ violates the GRS-condition, there exists $X=(x, \xi) \in \mathbb{R}^{2 d}$ such that $\lim _{n \rightarrow \infty} v(n X)^{1 / n}=$ $a=e^{\alpha}>1$. Let $\delta<\alpha / 2$ and set $A=\mathrm{I}-e^{-\delta} M_{\xi} T_{x}=\mathrm{I}-e^{-\delta} \pi(X)$. Then $A^{-1}=\sum_{n=0}^{\infty} e^{-n \delta} \pi(X)^{n}=\sum_{n=0}^{\infty} e^{-n \delta} c_{n} \pi(n X)$ for some coefficients $c_{n} \in \mathbb{C}$ with $\left|c_{n}\right|=1$. The symbol of $A$ is $\sigma(u, \eta)=1-e^{-\delta} e^{2 \pi i(u \cdot \xi+\eta \cdot x)} \in M_{v}^{\infty, 1}$, and the symbol $\tau$ of $A^{-1}$ is $\tau(u, \eta)=\sum_{n=0}^{\infty} e^{-n \delta} c_{n} e^{2 \pi i n(u \cdot \xi+x \cdot \eta)}$. One concludes by showing that $\tau \notin M_{v}^{\infty, 1}$. 


\section{Subconvolutive Weights}

Recall that a weight $v$ is subconvolutive, if $v^{-1} * v^{-1} \leq C v^{-1}$ and that $L_{v}^{\infty}$ consists of all functions satisfying the decay condition

$$
|f(t)| \leq C v(t)^{-1} .
$$

Subconvolutive weights are needed for the algebra property of decay conditions.

Lemma 7.1. The Banach space $L_{v}^{\infty}\left(\mathbb{R}^{d}\right)$ is a Banach algebra with respect to convolution, if and only if $v$ is subconvolutive.

Proof. If $f, g \in L_{v}^{\infty}$, then $|f(t)| \leq\|f\|_{L_{v}^{\infty}} v(t)^{-1}$ and $|g(t)| \leq\|g\|_{L_{v}^{\infty}} v(t)^{-1}$. Consequently,

$$
\mid(f * g)(t) \leq\|f\|_{L_{v}^{\infty}}\|g\|_{L_{v}^{\infty}}\left(v^{-1} * v^{-1}\right)(t) \leq C\|f\|_{L_{v}^{\infty}}\|g\|_{L_{v}^{\infty}} v^{-1}(t),
$$

and so $f * g \in L_{v}^{\infty}$. To obtain a Banach algebra norm, we endow $L_{v}^{\infty}$ with the equivalent norm $\|f\|_{L_{v}^{\infty}}^{\prime}=\sup _{h \in L_{v}^{\infty}}\|f * h\|_{L_{v}^{\infty}} /\|h\|_{L_{v}^{\infty}}$.

Conversely, assume that $\|f * g\|_{L_{v}^{\infty}} \leq C\|f\|_{L_{v}^{\infty}}\|g\|_{L_{v}^{\infty}}$ for all $f, g \in L_{v}^{\infty}$. Choosing $f=g=v^{-1}$, we find that $\left\|v^{-1}\right\|_{L_{v}^{\infty}}=1$ and $\left\|v^{-1} * v^{-1}\right\|_{L_{v}^{\infty}} \leq C\left\|v^{-1}\right\|_{L_{v}^{\infty}}=C$. Explicitly, this means that $\sup _{t \in \mathbb{R}^{d}}\left(v^{-1} * v^{-1}\right)(t) v(t) \leq C$, i.e., $v$ is subconvolutive.

7.1. Subconvolutive Weights in Time-Frequency Analysis. In time-frequency analysis subconvolutive weights and the corresponding modulation spaces $M_{v}^{\infty}$ are used sometimes as a substitute for the $M_{v}^{1}$-spaces. The condition $f \in M_{v}^{\infty}\left(\mathbb{R}^{d}\right)$ means that

$$
\left|V_{g} f(z)\right| \leq C \frac{1}{v(z)}
$$

Thus the condition $f \in M_{v}^{\infty}\left(\mathbb{R}^{d}\right)$ describes a genuine decay of $f$ in the timefrequency plane and is perhaps more intuitive than integrability conditions.

As an example for the occurrence of subconvolutive weights we state a theorem on Gabor frames taken from [15, Theorem 13.5.3] that parallels Theorem 6.9.

Theorem 7.2. Let $v_{s}(z)=(1+|z|)^{s}$ for $z \in \mathbb{R}^{2 d}$ and $s>2 d$. If $g \in M_{v_{s}}^{\infty}, \Lambda$ is a lattice in $\mathbb{R}^{2 d}$, and $\mathcal{G}(g, \Lambda)$ is a Gabor frame for $L^{2}\left(\mathbb{R}^{d}\right)$, then the (canonical) dual window $\gamma$ is also in $M_{v_{s}}^{\infty}$.

Since $M_{v_{s}}^{\infty}\left(\mathbb{R}^{d}\right) \subseteq M_{v_{s-2 d}}^{1}\left(\mathbb{R}^{d}\right)$, the time-frequency analysis of Theorem 6.11 holds for all modulation spaces $M_{m}^{p, q}$, where $m$ is a $v_{s-2 d}$-moderate weight.

Another application of subconvolutive weights is in the definition of time-frequency molecules [16].

Definition 7. A set $\left\{e_{z}: z \in \mathcal{Z}\right\}$ (for some discrete index set $\mathcal{Z} \subseteq \mathbb{R}^{2 d}$ ) is called a set of time-frequency molecules (of decay $s>2 d$ ), if

$$
\left|V_{g} e_{z}(w)\right| \leq C(1+|w-z|)^{-s} \quad \forall z \in \mathcal{Z}, w \in \mathbb{R}^{2 d} .
$$


This means that $e_{z}$ is centered near $z$ in the time-frequency plane and that all functions $e_{z}$, when shifted to the origin, possess a common time-frequency envelope.

The main theorem about time-frequency molecules is in the spirit of Theorem 6.9.

Theorem 7.3. Assume that $\left\{e_{z}: z \in \mathcal{Z}\right\}$ is a Gabor frames consisting of timefrequency molecules of decay $s>2 d$. Then the dual frame is again a set of timefrequency molecules of decay s.

REMARK: This theorem rests very much on theorems about inverse-closedness, and can also be formulated for $\ell^{1}$-conditions (as is shown in [3]) and for more general subconvolutive weights, namely precisely those satisfying the GRS-condition.

7.2. Pseudodifferential Operators. Subconvolutive weights occur in the definition of Sjöstrand's class with decay conditions. Let $v$ be a weight on $\mathbb{R}^{2 d}$ and define $M_{1 \otimes v}^{\infty}\left(\mathbb{R}^{2 d}\right)$ by the norm

$$
\|\sigma\|_{M_{1 \otimes v}^{\infty}\left(\mathbb{R}^{2 d}\right)}=\sup _{X, \Xi \in \mathbb{R}^{2 d}}\left|V_{\Phi} \sigma(X, \Xi)\right| v(\Xi) .
$$

The following theorem is a variation of Theorem 6.15, see [22].

Theorem 7.4. Assume that $v$ is subconvolutive, moderate on $\mathbb{R}^{2 d}$ and satisfies the $G R S$-condition. If $\sigma \in M_{1 \otimes v}^{\infty}$ and the corresponding pseudodifferential operator $K_{\sigma}$ is invertible on $L^{2}\left(\mathbb{R}^{d}\right)$, then there exists a symbol $\tau \in M_{1 \otimes v}^{\infty}$, such that $K_{\tau}=K_{\sigma}^{-1}$. In other words, the algebra $\mathrm{Op}\left(M_{1 \otimes v}^{\infty}\right)$ is inverse-closed in $\mathcal{B}\left(L^{2}\left(\mathbb{R}^{d}\right)\right)$.

\section{Beurling-Domar Weights}

A weight $v$ on $\mathbb{R}^{d}$ is said to satisfy the Beurling-Domar condition $[4,9,37]$, if

$$
\sum_{n=0}^{\infty} \frac{\log v(n x)}{n^{2}}<\infty \quad \forall x \in \mathbb{R}^{d} .
$$

Like the GRS-condition, this condition describes a form of subexponential decay and excludes weights having exponential growth. However, there is a fine line between GRS-weights and BD-weights. The weight $e^{|x| / \log (e+|x|)}$ satisfies the GRScondition, but not the BD-condition.

Many constructions and proofs in analysis employ localization techniques. This means that a property is first proved for functions with compact support and then extended to all functions in a space either by density or by an argument using a partition of unity. Obviously this method requires the existence of test functions with compact support.

BD-weights characterize the existence of test functions with compact support.

Given a submultiplicative weight on $\mathbb{R}^{d}$, we look at the image of $L_{v}^{1}\left(\mathbb{R}^{d}\right)$ under the Fourier transform. Formally the Beurling algebra is defined as

$$
\mathcal{F} L_{v}^{1}\left(\mathbb{R}^{d}\right)=\left\{f: f=\hat{h} \text { for some } h \in L_{v}^{1}\left(\mathbb{R}^{d}\right)\right\} .
$$


The norm is $\|f\|_{\mathcal{F} L_{v}^{1}}=\left\|\mathcal{F}^{-1} f\right\|_{L_{v}^{1}}$. With this norm, $\mathcal{F} L_{v}^{1}$ is a commutative Banach algebra with respect to pointwise multiplication. The underlying question is whether $\mathcal{F} L_{v}^{1}$ admits functions of arbitrarily small compact support. This question is rather subtle, and the existence of functions with compact support is not granted automatically. For instance, assume that $v(x)=e^{a|x|}$ is an exponential weight. By a theorem of Paley and Wiener the Fourier transform of any $h \in L_{v}^{1}$ can be extended to an analytic function on the strip $\{z \in \mathbb{C}:|\Im z|<a\}$. Thus the Beurling algebra $\mathcal{F} L_{v}^{1}$ cannot contain any functions with compact support.

A famous theorem of Beurling [4] provides a complete characterization of those algebras that contain functions with small compact support.

Theorem 8.1. Let $v$ be a submultiplicative (and continuous) weight on $\mathbb{R}^{d}$. The following conditions are equivalent:

(i) $\mathcal{F} L_{v}^{1}\left(\mathbb{R}^{d}\right)$ contains functions with arbitrarily small support, i.e., for every $\epsilon>0$ there is an $f \in \mathcal{F} L_{v}^{1}$ with supp $f \subseteq[-\epsilon, \epsilon]^{d}$.

(ii) The weight $v$ satisfies the logarithmic integral condition

$$
\int_{\mathbb{R}^{d}} \frac{\log v(x)}{(1+|x|)^{d+1}} d x<\infty .
$$

(iii) $v$ satisfies the Beurling-Domar condition

$$
\sum_{n=0}^{\infty} \frac{\log v(n x)}{n^{2}}<\infty \quad \forall x \in \mathbb{R}^{d} .
$$

REMARK: In contrast to condition (ii), the condition of Domar (46) can be formulated on arbitrary groups. The equivalence $(i) \Leftrightarrow($ iii $)$ holds for arbitrary locally compact abelian groups [9].

In time-frequency analysis we may ask an analogous question about the modulation spaces $M_{v}^{1}$ because these are the preferred spaces of test functions. Indeed we have the following statement [24].

Theorem 8.2. Let $v$ be a submultiplicative weight on $\mathbb{R}^{2 d}$ and assume that $v(x, \xi) \leq$ $C v(\xi,-x)$. The following conditions are equivalent:

(i) The modulation space $M_{v}^{1}$ contains functions of arbitrarily small compact support.

(ii) $M_{v}^{1}$ contains functions whose Fourier transform has arbitrarily small compact support.

(iii) $v$ satisfies satisfies the logarithmic integral condition

$$
\int_{\mathbb{R}^{2 d}} \frac{\log v(z)}{(1+|z|)^{2 d+1}} d z<\infty .
$$

(iv) $v$ satisfies the Beurling-Domar condition.

In several early papers on time-frequency analysis the BD-condition was assumed as the standard condition on the class of weights. In retrospect, this condition is often stronger than what is needed, but the existence of test functions with compact support is certainly natural and convenient. 
We note that this requirement is not satisfied for all spaces of test functions that arise in time-frequency analysis. For instance, if $v(z)=e^{a|z|}, a>0$, then the BD-condition is violated, and so $M_{v}^{1}$ does not contain functions with compact support or bandlimited functions. Likewise the universal space of test functions that works for all weights, the Gelfand-Shilov space $S^{1 / 2,1 / 2}$ proposed in [8] or the space of special windows $\mathcal{S}_{\mathcal{C}}$ defined in (28) do not contain functions with compact support. Thus several standard constructions of analysis, such as the construction of bounded uniform partitions of unity, cannot be carried out in these spaces.

\section{Other Classes of Weights and Further Remarks}

In harmonic analysis several other classes of weight function are encountered. As a class of particular interest we mention the Muckenhaupt weights. This characterize the validity of certain weighted norm inequalities, e.g., for the Hardy-Littlewood function or for the Fourier transform. However, in general Muckenhaupt weights are not moderate, therefore weighted $L^{p}$-spaces with respect to Muckenhaupt weights are not translation invariant. Consequently, the weighted modulation spaces $M_{m}^{p, q}$ where $m$ is a Muckenhaupt weight are not invariant under time-frequency shifts and thus do not fall under the realm of time-frequency analysis.

A first and fascinating application of Muckenhaupt weights in time-frequency analysis was found by Heil and Powell [25] in their investigation of Gabor systems at the critical density. They proved that the Gabor system $\mathcal{G}\left(g, \mathbb{Z}^{2}\right)$ is a Schauder basis for $L^{2}(\mathbb{R})$ for some enumeration of $\mathbb{Z}^{2}$, if and only if the Zak transform of $g$ is in the Muckenhaupt class $A_{2}\left(\mathbb{T}^{2}\right)$.

\section{REFERENCES}

[1] W. Arveson. Discretized CCR algebras. J. Operator Theory, 26(2):225-239, 1991.

[2] R. Balan. A noncommutative Wiener lemma and a faithful tracial state on Banach algebra of time-frequency operators. Trans. Amer. Math. Soc., 2007. To appear.

[3] R. Balan, P. G. Casazza, C. Heil, and Z. Landau. Density, overcompleteness, and localization of frames. II. Gabor systems. J. Fourier Anal. Appl., 12(3):309-344, 2006.

[4] A. Beurling. Sur les intégrales de Fourier absolument convergentes. In IX Congrès Math. Scand., pages 345-366, Helsinki, 1938.

[5] G. Björk. Linear partial differential operators and generalized distributions. Ark. Mat., 6:351407, 1966.

[6] A. Connes. $C^{*}$ algèbres et géométrie différentielle. C. R. Acad. Sci. Paris Sér. A-B, 290(13):A599-A604, 1980.

[7] E. Cordero and K. Gröchenig. Symbolic calculus and Fredholm property for localization operators. J. Fourier Anal. Appl., 12(4):345-370, 2006.

[8] E. Cordero, S. Pilipović, N. Teofanov, and L. Rodino. Localization operators and exponential weights for modulation spaces. Mediterr. J. Math. 2, pages 381-394, 2005.

[9] Y. Domar. Harmonic analysis based on certain commutative Banach algebras. Acta Math., 96:1-66, 1956.

[10] N. Dunford and J. T. Schwartz. Linear operators. Part I. Wiley Classics Library. John Wiley \& Sons Inc., New York, 1988. General theory, With the assistance of William G. Bade and Robert G. Bartle, Reprint of the 1958 original, A Wiley-Interscience Publication.

[11] H. G. Feichtinger. Gewichtsfunktionen auf lokalkompakten Gruppen. Österreich. Akad. Wiss. Math.-Natur. Kl. Sitzungsber. II, 188(8-10):451-471, 1979. 
[12] H. G. Feichtinger and K. Gröchenig. Banach spaces related to integrable group representations and their atomic decompositions. I. J. Functional Anal., 86(2):307-340, 1989.

[13] G. Fendler, K. Gröchenig, and M. Leinert. Symmetry of weighted $L^{1}$-algebras and the GRScondition. Bull. London Math. Soc., 38(4):625-635, 2006.

[14] I. Gel'fand, D. Raikov, and G. Shilov. Commutative normed rings. Chelsea Publishing Co., New York, 1964.

[15] K. Gröchenig. Foundations of time-frequency analysis. Birkhäuser Boston Inc., Boston, MA, 2001.

[16] K. Gröchenig. Localization of frames, Banach frames, and the invertibility of the frame operator. J.Fourier Anal. Appl., 10(2), 2004.

[17] K. Gröchenig. Composition and spectral invariance of pseudodifferential operators on modulation spaces. J. Anal. Math., 98:65 - 82, 2006.

[18] K. Gröchenig. Time-frequency analysis of Sjöstrand's class. Revista Mat. Iberoam., 22(2):703724, 2006. arXiv:math.FA/0409280v1.

[19] K. Gröchenig and M. Leinert. Wiener's lemma for twisted convolution and Gabor frames. J. Amer. Math. Soc., 17:1-18, 2004.

[20] K. Gröchenig and M. Leinert. Symmetry and inverse-closedness of matrix algebras and symbolic calculus for infinite matrices. Trans. Amer. Math. Soc., 358:2695-2711, 2006.

[21] K. Gröchenig and Y. Lyubarskii. Gabor frames with Hermite functions. Preprint, 2006.

[22] K. Gröchenig and Z. Rzeszotnik. Almost diagonalization of pseudodifferential operators. Preprint, 2006.

[23] K. Gröchenig and T. Strohmer. Analysis of pseudodifferential operators of sjöstrand's class on locally compact abelian groups. J. Reine Angew. Math. To appear.

[24] K. Gröchenig and G. Zimmermann. Spaces of test functions via the stft. J. Function Spaces Appl., 2(1):25-53, 2004.

[25] C. Heil and P. Alex. Gabor Schauder bases and the Balian-Low theorem. J. Math. Phys., 2007. To appear.

[26] L. Hörmander. The analysis of linear partial differential operators. I. Springer-Verlag, Berlin, second edition, 1990. Distribution theory and Fourier analysis.

[27] L. Hörmander. The analysis of linear partial differential operators. III, volume 274 of Grundlehren der Mathematischen Wissenschaften [Fundamental Principles of Mathematical Sciences]. Springer-Verlag, Berlin, 1994. Pseudo-differential operators, Corrected reprint of the 1985 original.

[28] A. J. E. M. Janssen. Signal analytic proofs of two basic results on lattice expansions. Appl. Comput. Harmon. Anal., 1(4):350-354, 1994.

[29] A. J. E. M. Janssen. Duality and biorthogonality for Weyl-Heisenberg frames. J. Fourier Anal. Appl., 1(4):403-436, 1995.

[30] H. Komatsu. Ultradistributions. I. Structure theorems and a characterization. J. Fac. Sci. Univ. Tokyo Sect. IA Math., 20:25-105, 1973.

[31] P. Koosis. The logarithmic integral. I. Cambridge University Press, Cambridge, 1998. Corrected reprint of the 1988 original.

[32] F. Luef. On spectral invariance of non-commutative tori. In Operator Theory, Operator Algebras, and Applications, volume 414, pages 131-146. American Mathematical Society, 2006.

[33] Y. I. Lyubarskil. Frames in the Bargmann space of entire functions. In Entire and subharmonic functions, pages 167-180. Amer. Math. Soc., Providence, RI, 1992.

[34] M. A. Nalmark. Normed algebras. Wolters-Noordhoff Publishing, Groningen, third edition, 1972. Translated from the second Russian edition by Leo F. Boron, Wolters-Noordhoff Series of Monographs and Textbooks on Pure and Applied Mathematics.

[35] T. W. Palmer. Banach algebras and the general theory of ${ }^{*}$-algebras. Vol. I, volume 49 of Encyclopedia of Mathematics and its Applications. Cambridge University Press, Cambridge, 1994. Algebras and Banach algebras. 
[36] S. Pilipović and N. Teofanov. Pseudodifferential operators on ultra-modulation spaces. J. Funct. Anal., 208(1):194-228, 2004.

[37] H. Reiter. Classical harmonic analysis and locally compact groups. Clarendon Press, Oxford, 1968.

[38] M. A. Rieffel. Projective modules over higher-dimensional noncommutative tori. Canad. J. Math., 40(2):257-338, 1988.

[39] K. Seip. Density theorems for sampling and interpolation in the Bargmann-Fock space. I. J. Reine Angew. Math., 429:91-106, 1992.

[40] J. Sjöstrand. An algebra of pseudodifferential operators. Math. Res. Lett., 1(2):185-192, 1994.

[41] J. Sjöstrand. Wiener type algebras of pseudodifferential operators. In Séminaire sur les Équations aux Dérivées Partielles, 1994-1995, pages Exp. No. IV, 21. École Polytech., Palaiseau, 1995.

[42] J. Toft. Subalgebras to a Wiener type algebra of pseudo-differential operators. Ann. Inst. Fourier (Grenoble), 51(5):1347-1383, 2001.

Faculty of Mathematics, University of Vienna, Nordbergstrasse 15, A-1090 ViENNA, Austria

E-mail address: karlheinz.groechenig@univie.ac.at 